in patients with advanced gastrointestinal stromal tumour after failure of imatinib: a randomised controlled trial. Lancet. 368:1329-1338.

7. Desai, J., et al. 2007. Clonal evolution of resistance to imatinib in patients with metastatic gastrointestinal stromal tumors. Clin. Cancer Res. 13:5398-5405.

8. Talpaz, M., et al. 2006. Dasatinib in imatinib-resistant Philadelphia chromosome-positive leukemias. N. Engl. J. Med. 354:2531-2541.

9. Kerkela, R., et al. 2006. Cardiotoxicity of the cancer therapeutic agent imatinib mesylate. Nat. Med. 12:908-916
10. Force, T., Krause, D.S., and Van Etten, R.A. 2007. Molecular mechanisms of cardiotoxicity of tyrosine kinase inhibition. Nat. Rev. Cancer. 7:332-344.

11. Verweij, J., et al. 2007. Imatinib does not induce cardiac left ventricular failure in gastrointestinal stromal tumours patients: Analysis of EORTC-ISGAGITG study 62005. Eur. J. Cancer. 43:974-978.

12. Khakoo, A.Y., et al. 2007. Rare incidence of congestive heart failure (CHF) in gastrointestinal stromal tumor (GIST) and other sarcoma patients (pts) receiving imatinib mesylate (IM) therapy. In ASCO Annual Meeting Proceedings Part I. Vol.
25, no. $18 \mathrm{~S}$ (June 20 supplement). J. Clin. Oncol. 2007:10026.

13. Fernández, A., et al. 2007. An anticancer CKit kinase inhibitor is reengineered to make it more active and less cardiotoxic. J. Clin. Invest. 117:4044-4054.

14. Hodi, F.S., et al. 2007. Major response to imatinib mesylate in KIT mutated melanoma. J. Clin. Oncol. In press.

15. Ostman, A., and Heldin, C.H. 2007. PDGF receptors as targets in tumor treatment. Adv. Cancer Res. 97:247-274.

\title{
The weight of cell identity
}

\author{
David T. Scadden
}

Center for Regenerative Medicine, Massachusetts General Hospital, Boston, Massachusetts, USA. Harvard Stem Cell Institute and Department of Stem Cell and Regenerative Biology, Harvard University, Cambridge, Massachusetts, USA.

\begin{abstract}
Recent studies involving molecular modification of adult somatic cells have pointed to a remarkable plasticity in cell identity. In this issue of the JCI, Koh and colleagues assessed whether bone marrow-derived cells could alter their fate under circumstances conducive to adipocyte generation in vivo (see the related article beginning on page 3684). These cells remained true to their roots, indicating how difficult it will be to exploit cell plasticity for therapeutic purposes.
\end{abstract}

Fat, the ever-popular source of personal and now societal anxiety, is of great scientific interest as a multidimensional regulator of health and longevity. Its origins have, like many cells of mesenchymal lineage, been explored to only limited extent. Progenitor cells of adipose tissue, bone, and cartilage are thought to descend from mesodermally derived multipotential mesenchymal stem cells (MSCs). The presence of MSCs in adult tissues has been demonstrated in mice and humans, but how they contribute to the maintenance or repair of adult tissues has not been defined, in part due to the lack of MSC-specific promoters that would enable lineage-tracking experiments. Where MSCs reside within tissues was also obscure; therefore, tracking these cells by in situ labeling was also difficult. Now it appears they reside in a perivascular location (1). Similarly, the developmental origin of MSCs has not been well characterized and is presumed to be mesodermal, but new information indicates that some MSCs derive from the neural crest (2).

Nonstandard abbreviations used: iPC, induced pluripotent cell; MSC, mesenchymal stem cell.

Conflict of interest: The author is a stockholder in Fate Therapeutics Inc.

Citation for this article: J. Clin. Invest. 117:3653-3655 (2007). doi:10.1172/JCI34181.

\section{Adipogenesis and adipocyte progenitor cells}

Adipocytes of both brown and white fat are thought to descend from MSCs by poorly defined intermediates and can respond in activity and number to a range of different stimuli. Adipocytes can, perhaps unfortunately, regenerate in settings of destruction and do have the capacity to transition between brown and white adipocytes. Whether they increase in number by proliferation as mature cells, amplification of precursor cell populations, or development from MSCs is not clear. Recently, it was reported by Crossno et al. that, in mice, adipocytes may derive from blood-borne cells under specific conditions: a high-fat diet or treatment with the antidiabetic drug rosiglitazone (3). In this issue of the JCI, that conclusion is drawn into question by studies of similar design reported by Koh et al. (4). In both studies $(3,4)$ bone marrow containing a genetic tracer (GFP) was transplanted from a donor mouse into a recipient mouse. The contribution of the donor bone marrow-derived cells to the adipocyte population was then assessed in the recipient animals. The analytical methods used in the two studies were different. Greater singlecell precision via the use of microscopy was achieved in the Koh et al. study, suggesting that evidence of bone marrow-derived cell contributions to adipocytes reported by Crossno et al. (3) was perhaps the result of inadequate discrimination of adipocytes from neighboring macrophages. In contrast, Crossno et al. examined GFP-positive adipocytes by flow cytometry and could detect no hematopoietic markers (CD45 and CD11b) in GFP-positive adipocytes. Since macrophages in fat do phagocytose adipocytes and become multinucleated, it may be that the fused cells have lost detectable CD45 or CD11b and yet continue to have detectable levels of GFP. With the new data reported in this issue, it is increasingly difficult to accept the alternatives that bone marrow-derived mesenchymal precursors form distant adipocytes or that adipocytes have the ability to be generated by hematopoietic cell populations.

\section{Fate specification and plasticity}

The notion that cells might take on an alternative fate even after the specification of identity has been an intriguing concept for some time, with waxing and waning evidence inspiring debate. A change in cellular fate might occur through two basic processes: conversion from one mature cell type to another, called transdifferentiation; or reversion to a less-differentiated state with subsequent maturation along alternative cell lineages, called dedifferentiation. Each of these processes would involve a reprogramming process that presupposes a great deal of plasticity in the mature cell.

That these processes can occur experimentally was defined decades ago by John Gurdon's work transferring the nucleus of a Xenopus enterocyte into an enucleated Xenopus oocyte (5). In that setting, he 
showed that the enterocyte nucleus led to development of a fully developed, mature, and fertile individual organism. This was clear evidence that cell identity, often considered an inexorable and unidirectional process from precursor to mature cell, retained a great deal of inherent plasticity. What was less clear was whether this was a curiosity that might have little relevance to mammals, somewhat akin to the ability of the newt to regrow severed limbs. That comfortable notion was exploded in 1996 when Ian Wilmut and colleagues reported on Dolly, the sheep cloned by nuclear transfer from a cultured cell line (6). This study revealed that nuclei from somatic cells could be induced to revert back to an embryonic state by poorly understood processes occurring in and mediated by the cytoplasm of the unfertilized egg.

\section{Reprogramming somatic cells}

In the last year, the mysterious ability of egg cytoplasm to rewind a cell's developmental history has become a tractable biochemical process through the groundbreaking work of Shinya Yamanaka and colleagues. They documented, and others have extensively validated, that fibroblasts from a mouse's tail could be "pushed back" to a fully functional ES cell state with the introduction of four specific cDNAs (7-9). Quite remarkably, mature cells were demonstrated to change their functional phenotype, gene expression profile, and even epigenetic signature to exactly mimic ES cells. These induced pluripotent cells (iPCs) were capable of yielding chimeric animals when iPCs were injected into blastocysts, and the iPCs contributed to all cell types, including germ cells. Plasticity of somatic cells thereby transitioned from a curiosity to an approachable molecular biology puzzle that has triggered enormous interest in defining: (a) whether the same feat can be accomplished with human cells; (b) whether all somatic cells can be dedifferentiated; (c) whether other cDNAs can substitute for the original four introduced (Oct3/4, Sox2, $K l f 4$, and $c-m y c$ ); (d) what epigenetic changes occur sequentially to enable this reversion in fate; and, finally, (e) whether small molecules or other exogenous factors can induce this dramatic change in cell state. The latter is of great interest from a therapeutic perspective because it might lead to a drug-based approach for the conversion, for example, of a cell in a terminally differentiated, postmitotic state to one in a progenitor-like state. One consequence of such an approach might be the ability to regenerate normal tissues in settings of disease or injury.

Transitioning back to a progenitor state might require less biochemical alteration than dedifferentiation fully back to a pluripotent stem cell. For example, a single genetic manipulation was recently shown to accomplish this. Cobaleda and colleagues demonstrated that the paired box gene 5 (Pax5) gene necessary for $\mathrm{B}$ cell differentiation is also required for the maintenance of mature B cells (10). When Pax5 was conditionally deleted in adult mice, the mature B cells reverted to a common lymphoid precursor phenotype. These cells were then able to differentiate into a spectrum of $\mathrm{T}$ lymphocyte precursors and mature $\mathrm{T}$ cells. Thus, mature B cell dedifferentiation could occur in vivo with altered expression of a single gene product. Others had previously shown that mature B cells could transdifferentiate to cells of a monocyte fate with manipulation of other gene products: CCAAT/enhancer-binding proteins $\alpha$ and $\beta$ (11). Taken together, these data now raise the question of whether certain conditions, perhaps even those encountered with altered physiologic settings, might themselves be capable of dedifferentiating or transdifferentiating cells in vivo.

\section{Reprogramming in vivo}

This conversion of cell fates in vivo is well documented in invertebrates. For example, in Drosophila, when maturing daughter cells of germ cell stem cells come in contact with the stem cell niche, the cells reacquire a stem cell phenotype (12). In vertebrates, injury to tissues of teleost fish (such as zebrafish) or urodele amphibians (such as salamanders) results in dedifferentiation of cells to a blastema that then recapitulates a developmental program resulting in tissue regeneration (13). But in mammals, the process of cells converting from one state to another is generally associated with very limited circumstances. For example, epithelial-to-mesenchymal transition occurs in development and in malignancy. Whether similar types of conversions might occur in other contexts is the issue that makes studies such as that reported in this issue of JCI by Koh and colleagues of interest (4).

Five to seven years ago, there were multiple reports of putative transdifferentiation of mammalian cells (14). These observations have since been largely reinterpreted as being due to the unexpected fusion of mature cells and not the result of transdif- ferentiation (15). Fusion of heterologous cells has been found to often result in the expression of an indicator gene from one fusing cell in the background of tissuespecific genes from the fusion partner. For example, a $\beta$-galactosidase-positive hepatocyte might initially be interpreted as being the result of hematopoietic cell transdifferentiation and then found instead to represent the fusion of a bone marrow-derived cell expressing $\beta$-galactosidase and a liver cell. When this fusion phenomenon was recognized, the results of many such studies reporting successful transdifferentiation were dismissed, along with the notion of transdifferentiation itself. Now that plasticity has been shown to exist as an experimentally inducible phenomenon, careful in vivo studies such as those by Koh and colleagues become of renewed importance (4).

In the current study (4), Young Jun Koh and colleagues in the laboratory of Gou Young Koh examined whether bone marrow-derived progenitor cells in the circulation contribute to adipogenesis via use of both the transplant model reported by Crossno et al. (3) and, in some experiments, parabiosis. This model joins the circulation of a GFP-positive transplanted mouse with the circulation of a control mouse by surgically connecting skin flaps. It has been very useful in examining patterns of cell migration and identifying cell sources (16). Koh et al. then examined whether the GFP-positive bone marrow-derived cells contributed in any way to adipose tissue. Importantly, they examined this under a variety of conditions, including wounding, drug treatment with rosiglitazone or granulocyte colony-stimulating factor, or feeding of a high-fat diet. Use of a range of nonhomeostatic settings is particularly important if we are to define whether cell fate changes can occur based on specific cell environments. Under no circumstance did the authors find GFP-expressing adipocytes in the recipient animals, despite finding mature hematopoietic cells (macrophages) in fat tissue. If the authors had found GFPpositive adipocytes, it is not clear how they could have distinguished transdifferentiation from adipogenic differentiation of circulating MSCs; but having found no GFPpositive adipocytes, it was reasonable to conclude that blood-borne contribution to adipogenesis simply does not happen with detectable frequency in these animals.

The issue of cell plasticity in mammals therefore appears to remain a largely laboratory-induced phenomenon. That cells can 
change their fate is clear. Whether they do it under physiologic conditions seems progressively less likely. Inducing cells to trans- or dedifferentiate by specific manipulations in vivo would open the possibility of generating specific types of new cells and be of great interest for the field of regenerative medicine. At present, it remains an unfulfilled challenge worthy of continual investigation.

Address correspondence to: David T. Scadden, Massachusetts General Hospital Center for Regenerative Medicine, 185 Cambridge Street, CPZN-4265A, Boston, Massachusetts 02114, USA. Phone: (617) 726-5615; Fax: (617) 726-4691; E-mail: scadden.david@ mgh.harvard.edu.

1. Sacchetti, B., et al. 2007. Self-renewing osteoprogenitors in bone marrow sinusoids can organize a hematopoietic microenvironment. Cell. 131:324-336.
2. Takashima, Y., et al. 2007. Neuroepithelial cells supply an initial transient wave of MSC differentiation. Cell. 129:1377-1388.

3. Crossno, J.T., Jr., Majka, S.M., Grazia, T., Gill, R.G., and Klemm, D.J. 2006. Rosiglitazone promotes development of a novel adipocyte population from bone marrow-derived circulating progenitor cells. J. Clin. Invest. 116:3220-3228.

4. Koh, Y.J., et al. 2007. Bone marrow-derived circulating progenitor cells fail to transdifferentiate into adipocytes in adult adipose tissues in mice. J. Clin. Invest. 117:3684-3695.

5. Gurdon, J.B. 1960. The developmental capacity of nuclei taken from differentiating endoderm cells of Xenopus laevis. J. Embryol. Exp. Morphol. 8:505-526.

6. Campbell, K.H., McWhir, J., Ritchie, W.A., and Wilmut, I. 1996. Sheep cloned by nuclear transfer from a cultured cell line. Nature. 380:64-66.

7. Takahashi, K., and Yamanaka, S. 2006. Induction of pluripotent stem cells from mouse embryonic and adult fibroblast cultures by defined factors. Cell. 126:663-676.

8. Okita, K., Ichisaka, T., and Yamanaka, S. 2007. Generation of germline-competent induced pluripotent stem cells. Nature. 448:313-317.

9. Wernig, M., et al. 2007. In vitro reprogramming of fibroblasts into a pluripotent ES-cell-like state. Nature. 448:318-324.

10. Cobaleda, C., Jochum, W., and Busslinger, M. 2007. Conversion of mature $\mathrm{B}$ cells into $\mathrm{T}$ cells by dedifferentiation to uncommitted progenitors. Nature. 449:473-477.

11. Xie, H., Ye, M., Feng, R., and Graf, T. 2004. Stepwise reprogramming of $\mathrm{B}$ cells into macrophages. Cell. 117:663-676.

12. Kai, T., and Spradling, A. 2004. Differentiating germ cells can revert into functional stem cells in Drosophila melanogaster ovaries. Nature. 428:564-569.

13. Harty, M., Neff, A.W., King, M.W., and Mescher, A.L. 2003. Regeneration or scarring: an immunologic perspective. Dev. Dyn. 226:268-279.

14. Lagasse, E., et al. 2000. Purified hematopoietic stem cells can differentiate into hepatocytes in vivo. Nat. Med. 6:1229-1234.

15. Wang, X., et al. 2003. Cell fusion is the principal source of bone-marrow-derived hepatocytes. Nature. 422:897-901.

16. Wagers, A.J., Sherwood, R.I., Christensen, J.L., and Weissman, I.L. 2002. Little evidence for developmental plasticity of adult hematopoietic stem cells. Science. 297:2256-2259.

\title{
OX40-OX40L interactions: a promising therapeutic target for allergic diseases?
}

\author{
Yui-Hsi Wang ${ }^{1}$ and Yong-Jun Liu'1,2
}

1Department of Immunology and Center of Cancer Immunology Research, The University of Texas MD Anderson Cancer Center, Houston, Texas, USA.

${ }^{2}$ The University of Texas Graduate School of Biomedical Sciences at Houston, Houston, Texas, USA.

Recent advances in understanding the cellular and molecular mechanisms of atopy have shed light on potential targets for the development of new therapies for allergic diseases. In this issue of the JCI, Seshasayee et al. provide direct in vivo evidence that $\mathrm{OX} 40$ has critical roles in allergic inflammation mediated by thymic stromal lymphopoietin (TSLP) (see the related article beginning on page 3868 ). Blockade of interactions between OX40 on Th2 cells and OX40 ligand (OX40L) on TSLP-activated DCs using an OX40L-specific monoclonal antibody, inhibited Th2 cell-mediated immune responses in both mouse and nonhuman primate models of allergic inflammation. The results point to potential therapeutic approaches to targeting the cellular and molecular mechanism underlying TSLP-mediated allergic inflammation.

\section{TSLP: bridging the epithelial barrier and type 2 immune responses}

Asthma and atopic dermatitis (AD) are inflammatory disorders characterized by the infiltration and accumulation of memory-like Th2 cells and eosinophils (1). In addition to the type 2 inflammatory processes, allergic diseases often involve epi-

Nonstandard abbreviations used: $\mathrm{AD}$, atopic dermatitis; OX40L, OX40 ligand; TSLP, thymic stromal lymphopoietin; TSLPR, TSLP receptor.

Conflict of interest: The authors have declared that no conflict of interest exists.

Citation for this article: J. Clin. Invest. 117:3655-3657 (2007). doi:10.1172/JCI34182. thelial cell stress and injury that trigger the release of chemokines and growth factors able to support both chronic inflammatory and remodeling responses. The idea that reduced barrier function of the epithelium and altered innate immunity are fundamental to the origin of these diseases is supported by the recent finding that thymic stromal lymphopoietin (TSLP), an IL-7-like cytokine, plays a key role in allergic inflammation at the interface between epithelial cells and DCs (2). TSLP is highly expressed by keratinocytes in skin lesions of patients with $\mathrm{AD}$ (3) and by airway epithelial cells of individuals with asthma (4) and can potently activate myeloid DCs by upregulating their surface expression of MHC class II, CD54, CD80, CD83, CD86, and DC-lamp (3). Interestingly, TSLP triggers human myeloid DCs to produce a myriad of chemokines that recruit eosinophils and Th 2 cells, but it does not trigger them to produce either Th1-polarizing cytokines of the IL-12 family or the proinflammatory cytokines TNF- $\alpha$, IL-1 $\beta$, and IL- 6 , resulting in a microenvironment permissive for type 2 inflammatory responses (5). TSLPactivated DCs can induce naive $T$ cells to differentiate into inflammatory Th2 cells producing the classical Th2 cytokines IL-4, IL-5, and IL-13, as well as a large amount of TNF- $\alpha$ but little or no IL-10 (6). Moreover, only TSLP-activated DCs can induce in vitro, allergen-specific Th2 memory cells to undergo homeostatic expansion and further Th2 polarization, and to mediate recall responses (7). In support of the findings in humans, mice with conditional overexpression of TSLP in keratinocytes were found to have an AD-like phenotype characterized by scaling lesions accompanied by infiltration of Th2 $\mathrm{CD}^{+}$cells in skin and elevated serum IgE level (8). By 